Produto \& Produção, vol. 11, n. 1, p. 30 - 40, fev. 2010

Edição Metrologia

\title{
Implementation of Double-Timing Pulse Interpolation Applied to Compact Piston Provers
}

\author{
Jeronymo, C.E. \\ INMETRO, Rio de Janeiro, Brazil, \\ cejeronymo@inmetro.gov.br \\ Araujo, S.B. \\ INMETRO, Rio de Janeiro, Brazil, \\ sbaraujo@inmetro.gov.br \\ Aibe, V.Y. \\ INMETRO, Rio de Janeiro, Brazil, \\ vyaibe@inmetro.gov.br
}

\begin{abstract}
This work presents an electronic circuit for double-timing pulse interpolation applied to compact piston provers (also referred as small volume provers). Compact provers are usually employed to prove meters with pulsed outputs. API and ISO standards [1,2] recommend a minimum of 10.000 pulses per run to obtain a resolution better than $\pm 0.01 \%$. Since the volume of fluid displaced by a compact prover is relatively small, the number of pulses produced during a proving run is often considerably less than 10.000 pulses. Pulse interpolation techniques are commonly used to increase resolution and to diminish uncertainty during a proving run by estimating the fractional part of meter pulses within the time interval of the calibration. In this way, pulse interpolation techniques are essential to obtain accurate flow measurements and to allow the calibration of meters with compact provers.

Our implementation uses a compact piston prover with an internal volume of $12 \mathrm{~L}$ and maximum flow capacity of $180 \mathrm{~L} / \mathrm{min}$. In order to implement the double-timing pulse interpolation method, we have used a Pentium D, 2.80 GHz installed with a 16bit counter/timer board. Data acquisition and control software were written using VB .Net. An electronic circuitry was built to activate/deactivate counters gates, and to collect pulses. Some requirements and limitations of pulse interpolation techniques such as circuitry testing and pulse stability are also discussed in this work.
\end{abstract}

Keywords: compact piston prover, pulse interpolation, measurement uncertainty, flowmeter calibration 


\section{INTRODUCTION}

Compact piston prover systems (also referred as small volume provers) are used in the calibration of flowmeters. They have long been accepted as primary flow calibrators for both gas and liquid flowmeters [1-3]. Basically they compare the volume of fluid displaced by a piston during a specific time interval with the volumetric flow measured by a meter [3]. Compact provers present many advantages compared to conventional provers, such as portability, low cost, use of small amounts of fluid, reduced time for calibrations and capacity of proving lower flow rates. Due to its low volume and limited number of pulses, it's of great importance to verify the pulse interpolation method is correctly specified and implemented. It's also important to observe that accuracy and applicability of pulse interpolation methods may deteriorate due to several factors, such as circuit configuration and pulse stability. The API and ISO standards recommend a minimum of 10,000 pulses collected per run $[1,2]$. Since the volume of fluid displaced by a compact prover is relatively small, most of the time it may not reach 10,000 pulses per run. In order to overcome this problem, pulse interpolation techniques are commonly used to estimate the fractional part of meter pulses within the time interval of the calibration [1-3]. Pulse interpolation techniques assume that flow is stable during a proving pass. ISO $7278-3$ establishes fluctuations in the flowrate during a pass must be limited to less than $\pm 2 \%$ of the mean flowrate.

The use of compact provers and interpolation techniques were formally recognized by API in 1988, with the publication of API MPMS, chapter 4. Nowadays, compact provers in conjunction with pulse interpolation techniques enjoy large acceptance in the industry. They are commonly utilized in petroleum platforms as a reliable and accurate instrument to calibrate meters in custody transfer applications. The Brazilian National Agency of Petroleum and Gas (ANP) through PANP/Inmetro $1 / 2000$ [4] establishes the regulation of the measuring system of petroleum and natural gas for production, transportation and storage in Brazil. According to the mentioned regulation, meter calibration systems for hydrocarbons can be done using different methods as long as they comply with ANP regulations. In the case of compact provers, which may collect a number of pulses limited to less than 10000 pulses, the ANP regulation establishes that ISO 7278-3 (Pulse Interpolation Techniques for Dynamic Measurements of Hydrocarbons) is applicable. Three different methods are described in the referred international standard: doubletiming, double-timing and phase-locked-loop techniques. Each method has its own particularities, hardware complexity and can be affected differently by flowrate fluctuations during a proving pass. The objective of this work is to implement the pulse interpolation technique double-timing (double chronometry). It's part of Inmetro's effort to disseminate interpolation techniques to the industry and improve calibration accuracy of meters in custody transfer applications.

There are different types of prover arrangements in the market [3,5-7]. The Inmetro Fluid Dynamics Metrology Division (Dinam) employs an active piston prover driven by compressed air. Figures 1 and 2 show schematically the working principle of this type of piston prover. Basically it has two operational modes: "run mode" and "return mode". During the "run mode" (Fig. 1), pressurized air is directed to chamber $B$ and the resultant force pushes the piston to the left at a constant speed. An incremental linear encoder connected to the piston rod detects the piston position and sends a pulse train digitizing the piston position to the computer. The linear 
encoder has a resolution of $10 \mu \mathrm{m}$ per pulse. At the same time, fluid is displaced from chamber $A$ and passes through the meter under test. The meter generates a pulse train proportional to the volume of fluid passing through it. Pulse train signals are processed in the computer and the displaced volume and correspondent flow rate is calculated. The volumetric relation between linear encoder pulses and piston traveled distance is previously determined using the water draw method or a master meter [2].

During the "return mode" the position of the control valve is diverted and pressurized air is sent to the top of the tank (Fig. 2). The piston moves to the right and chamber $A$ is completely filled with liquid. The system is then ready for a new calibration cycle.



Fig. 1. Schematic diagram of compact piston prover at the beginning of a calibration run

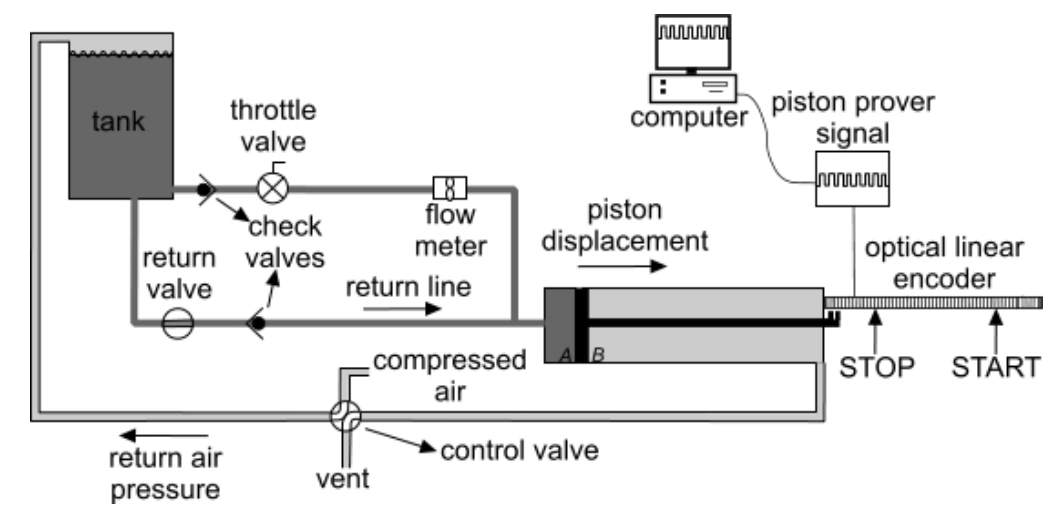

Fig. 2. Schematic diagram of compact piston prover at the beginning of return mode

\section{INTERPOLATION TECHNIQUES}

There are various techniques that can be used to implement pulse interpolation and estimate the fractional part of meter pulses within a time interval. Invariably they all require an electronic circuitry composed of high-speed timers, counters, control software and a microprocessor to execute the necessary calculation. The complexity of the hardware depends on the method being implemented. The ISO 7278-3 [1] acknowledges three interpolation techniques, i.e., double-timing, double-timing and phase-locked-loop. We explain below two of these techniques: double-timing and quadruple-timing interpolation. 
Figure 3 shows a schematic diagram of the double-timing method (also called double chronometry). In this figure, $T_{2}$ is the time interval containing a fractioned number of pulses $\left(n^{\prime}\right)$ between prover START and STOP detection signals and $T_{1}$ is the time interval containing the whole number of pulses $(n)$. Timer 1 stores the value of time $T_{1}$ and Timer 2 stores $T_{2}$.

The following sequence of events is employed in this method:

1. Start timer $2\left(T_{2}\right)$ when piston reaches START sensor.

2. Start timer $1\left(T_{1}\right)$ at the high edge of the next flow meter pulse following $T_{2}$ start.

3. Stop timer $2\left(T_{2}\right)$ when piston reaches STOP sensor.

4. Stop timer $1\left(T_{1}\right)$ at the high edge of the next flow meter pulse after $T_{2}$ stop.

The number of fractioned pulses $n^{`}$ within $T_{2}$, is obtained using the following equation:

$$
n^{`}=T_{2} \times n / T_{1}
$$

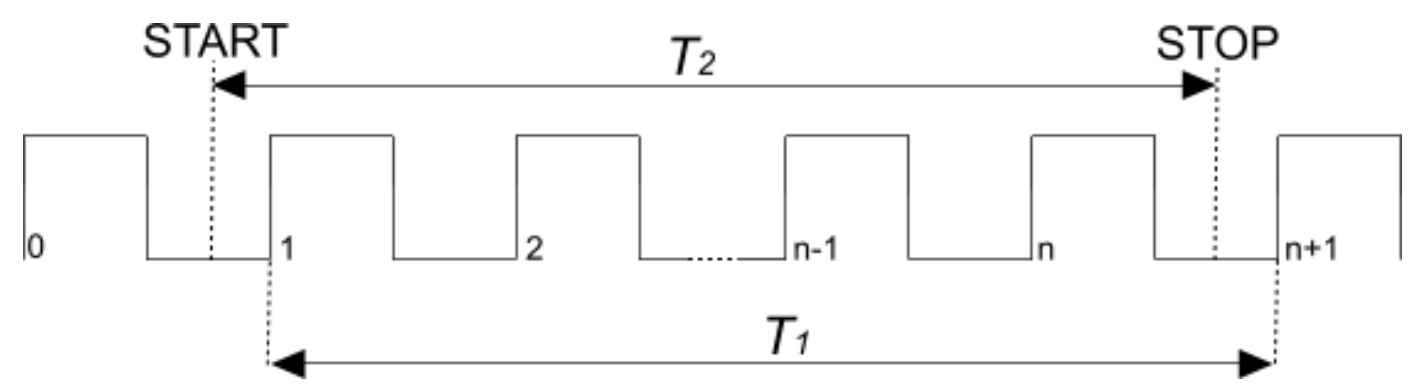

Fig. 3. Detection of meter pulses for the implementation of double-timing technique

\subsection{Quadruple-timing}

Figure 4 shows a schematic diagram of the quadruple-timing method. In this figure, $t$ is the time interval between START and STOP detection signals containing a fractioned number of pulses $n$. Times $t_{1}$ and $t_{3}$ are the time intervals of a fractioned pulse as being indicated on Fig. 4. Times $t_{2}$ and $t_{4}$ are the time intervals of a whole pulse as being indicated on Fig. 4. The number of interpolated pulses $n$ ' within $t$, is calculated using the following equation

$n^{`}=n+t_{1} / t_{2}-t_{3} / t_{4}$ 


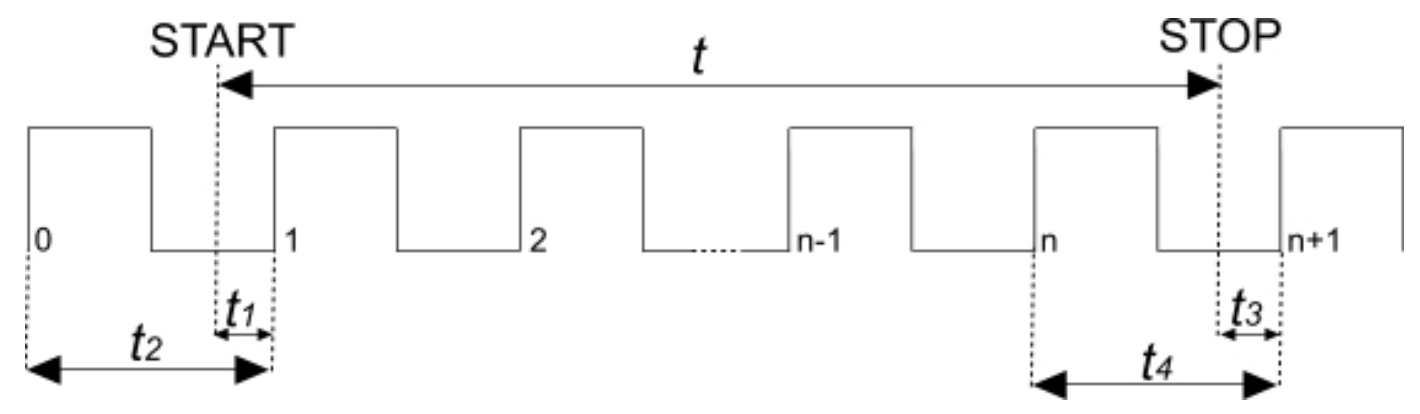

Fig. 4. Detection of meter pulses for the implementation of quadruple-timing technique

\section{IMPLEMENTED SYSTEM}

This work implements the double-timing technique. Our implementation uses a compact piston prover with an internal volume of $12 \mathrm{~L}$ and maximum flow capacity of $180 \mathrm{~L} / \mathrm{min}$. In order to implement the pulse interpolation method, we have used a Pentium D, 2.80 GHz installed with a high performance counter/timer board. The frequency of the internal clock of the board can be set from $10 \mathrm{kHz}$ to $5 \mathrm{MHz}$, much larger than required by ISO 7278-3 [1] for double-timing $(20 \mathrm{kHz})$ or for double-timing $(40 \mathrm{kHz})$. The data acquisition software was written using VB .Net. In order to measure the required time intervals and correspondent number of pulses, an electronic circuitry was built to collect pulses, activate/deactivate counter gates and interface with the control software.

A schematic diagram of the electronic circuit is shown in Fig. 5. The electronic circuit is composed of seven 16-bit counters. In this diagram counters are numbered from 1 to 7 , and schematically represented as having a Gate (gate-n), Counter Input (ctr-n) and Output Control (out-n). Initially all gates and outputs are at Low Level (LH). The JK flip-flop $[8,9]$ is reset before starting the data acquisition setting output $\mathrm{Q}_{2}$ to Low Level (LL). All counters are set to count pulses on failing edges. Counter 1 stores meter pulses, counter $2 \& 3$ are chained to form a 32 bit counter and store time $T_{2}$ (Fig. 3). Counter 4 stores pulses originating from the linear optical encoder. Counter 5 is an auxiliary counter used to define the piston position at which the pulse acquisition process will start, and counters 6 and 7 are chained to form a 32 bit counter and measure time $T_{1}$. An oscillator with a clock frequency of $1 \mathrm{MHz}$ is connected to Counter Input of counters 2 and 6. 


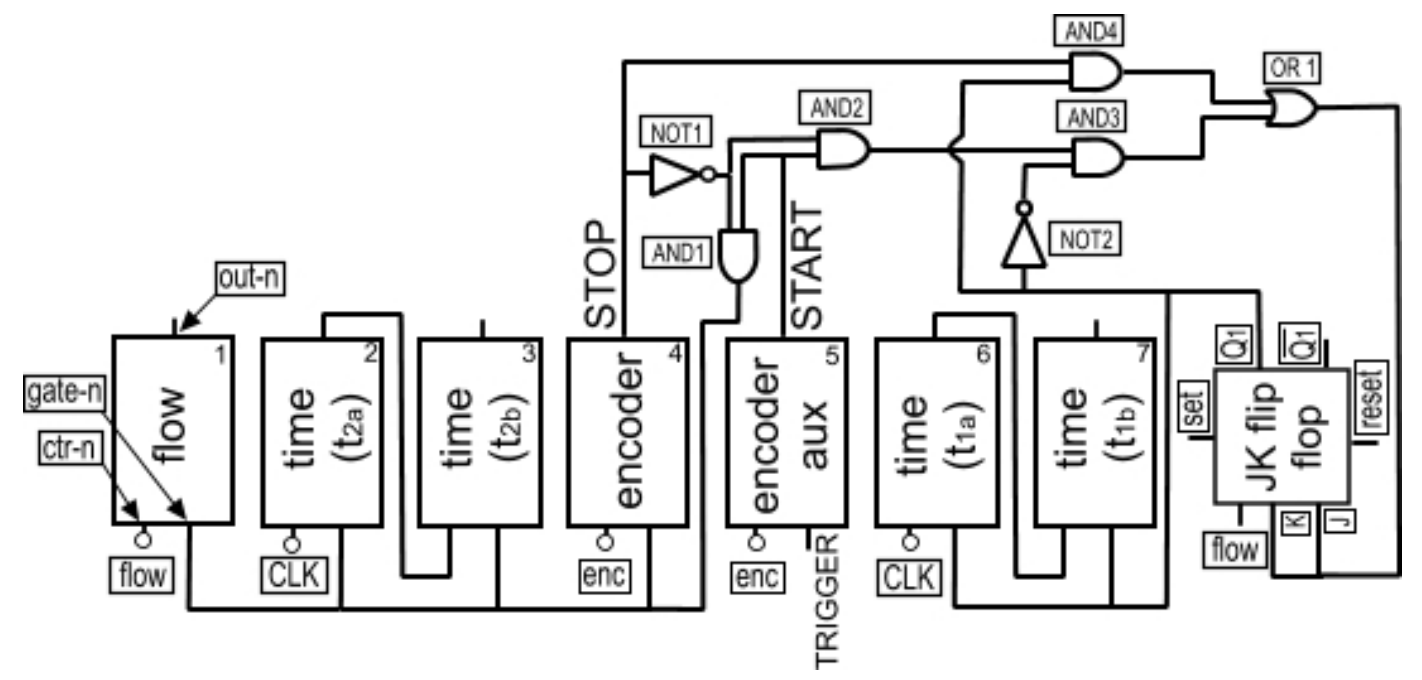

Fig. 5. Block diagram of pulse interpolation unit

Figure 6 illustrates the START/STOP process of the above circuitry. In this figure, $n_{\text {aux }}$ and $n_{\text {enc }}$ are user-defined parameters that can be set in the control program screen. After the user starts a new proving pass, Gate-5 (START) is set to High Level (HL). In this way Gate-5 is activated and counter 5 starts to collect encoder pulses. When counter 5 reaches $n_{\text {aux }}$ pulses, out- 5 becomes HL and gates1 (flow), $2 \& 3$ (time $T_{2}$ ) and 4 (encoder) are activated. Counter 4 starts collecting encoder pulses until $n_{\text {enc }}$ is reached. At this point out-4 turns High Level $(\mathrm{HL})$ deactivating gates-1, 2, 3, 4. Counter 1 has now stored the number of meter pulses $(n)$, counter 4 encoder pulses $n_{\text {enc }}$, and counters $2 \& 3$ the time duration of this process $\left(T_{2}\right)$. Note that piston traveled distance from START to STOP contains a whole number of linear encoder pulses since gate-4 is activated/deactivated at the exact position of an encoder pulse. This approach decreases the uncertainty in the calculation of the volumetric flow since the displaced volume is given by a whole number of encoder pulses. Since the number of accumulated encoder pulses defines volume of flow and time is known from counters $2 \& 3$, flow rate can also be calculated.

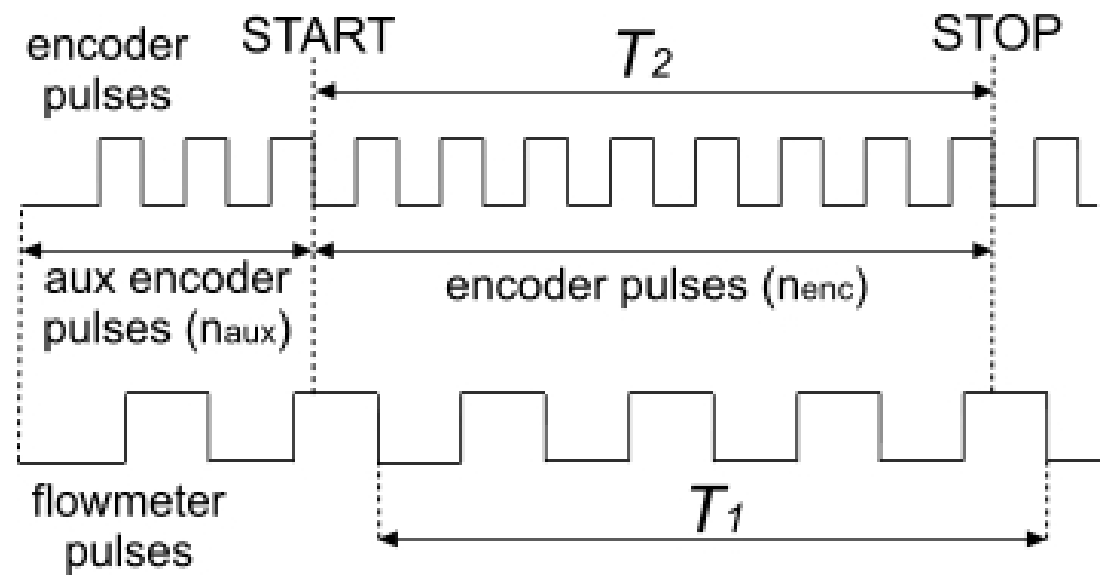

Fig. 6. Scheme for the testing of pulse interpolation unit 
Concerning the measurement of time $T_{1}$, the following approach is adopted. Initially output $Q_{1}$ of the JK flip-flop is at state low level and gates of counters 6 and 7 are deactivated. Ports Set and Reset are set to HL. Table 1 shows the function table of the flip-flop. Details about flip-flops circuits can be found in reference [8, 9]. When the output of auxiliary encoder out-5 is raised, the output of logical gate OR1 becomes $\mathrm{HL}$ and ports $\mathrm{J}$ and $\mathrm{K}$ turn $\mathrm{HL}$. The flip-flop turns to toggle mode. As soon as the first failing edge of the meter pulse is detected, output $Q_{1}$ turns $\mathrm{HL}$. Then output of NOT2 turns LL. Consequently output OR1 turns LL and flips flop ports $\mathrm{J}$ and $\mathrm{K}$ turn LL. The flip-flop is deactivated (see function Table 1) and output $Q_{1}$ is maintained $\mathrm{HL}$. At the end of the acquisition process out-4 (STOP) turns $\mathrm{HL}$ and the output of AND4 becomes HL. Output OR1 is now $\mathrm{HL}$ and the flip-flop is back to toggle mode. As soon as the first meter pulse lowering edge is detected, output $Q_{1}$ turns LL deactivating gates 6 and 7 . Counters 6 and 7 store now time $T_{1}$. The value of the interpolated pulses $n$ ' is calculated using Equation 1.

Table 1. Function table

\begin{tabular}{|c|c|c|c|c|c|c|}
\hline \multicolumn{5}{|c|}{ Inputs } & \multicolumn{2}{c|}{ Outputs } \\
\hline Set & Reset & Clock & J & K & Q & $\bar{Q}$ \\
\hline H & H & O & L & L & \multicolumn{2}{|c|}{ No change } \\
\hline H & H & O & H & H & \multicolumn{2}{|c|}{ Toggle } \\
\hline H & L & X & X & X & L & H \\
\hline
\end{tabular}

\section{TEST CIRCUIT}

ISO 7278-3, section 7.2, establishes requirements for testing a pulse interpolation circuitry and proposes a block diagram of a test circuit. Figure 7 shows a schematic diagram of the testing circuit we have used to check the response of the pulse interpolation electric circuitry we have proposed in section 3 . The testing circuit is composed of a pulse generator and a frequency divider, which allows us to generate two sets of pulses with different frequencies. The pulse train with higher frequency, $F$ drives a reference counter $A$, while the other pulse train with lower frequency $F / R$ drives the pulse interpolation unit under test. Both counters (counter $A$ and pulse interpolation unit) are controlled by simultaneous START/STOP signals and can be set to zero. As described by ISO 7278-3 [1], the readings in counter A have to agree with $n^{\prime} \times R$ within $0,01 \%$ for a time interval large enough to accumulate at least 10000 pulses. The input frequency can be constant $(\mathrm{dF} / \mathrm{dt}=0)$ or a ramp of variable frequencies $(\mathrm{dF} / \mathrm{dt} \neq 0)$. 


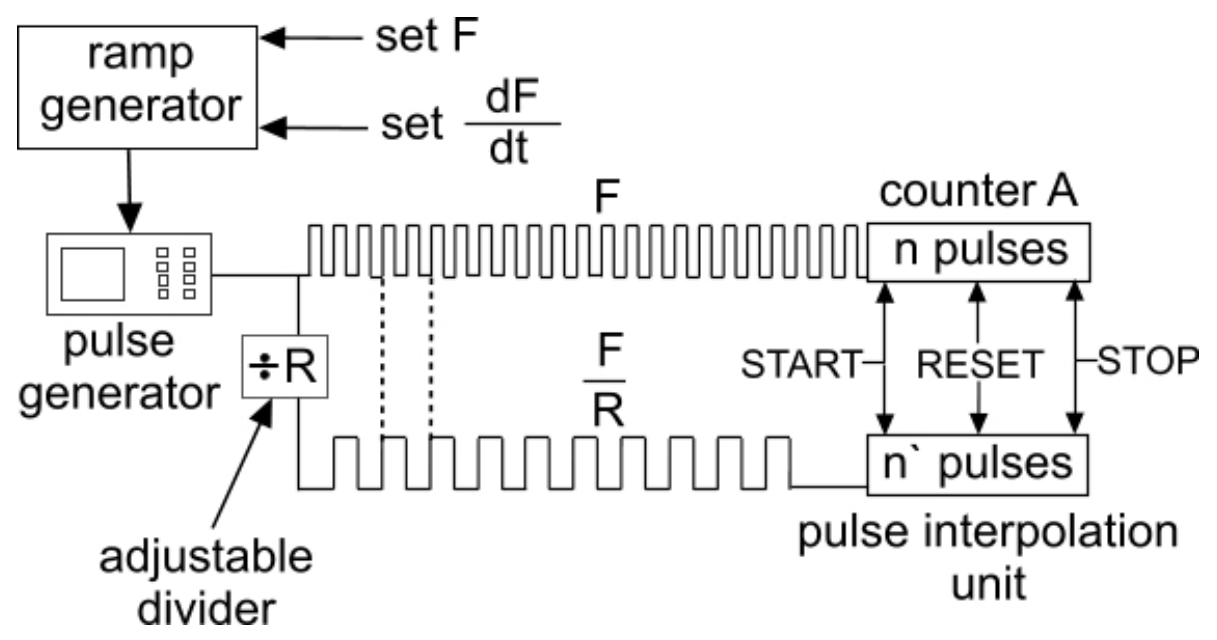

Fig. 7 Pulse interpolation testing

Tables 2, 3 show test results for constant pulse frequencies $(\mathrm{dF} / \mathrm{dt}=0)$. Table 2 shows results for a clock of $1 \mathrm{MHz}$ and Table 3 for a clock frequency of $100 \mathrm{kHz}$ (please note that the minimum clock recommend by API and ISO standards $[1,2]$ is $20 \mathrm{kHz})$. The interpolation error $\varepsilon(\%)$ is calculate using equation: $\varepsilon(\%)=100 \times\left(n^{\prime} \times R\right.$ $\mathrm{nA}) / \mathrm{nA}$. Both tables show the largest and the smallest errors obtained from a series of tests with $F / R$ frequencies of $10 \mathrm{kHz}(R=100), 1 \mathrm{kHz}(R=1000)$ and $0.5 \mathrm{kHz}$ $(R=2000)$. We can see that all test results have an error lower than $0.01 \%$. Also, as expected, we can see that error $\varepsilon$ decreases with a larger $R$ (lower F/R frequency) and increases with a lower clock frequency (100 kHz versus $1 \mathrm{MHz}$ clock).

Table 4 shows test results for $\mathrm{dF} / \mathrm{dt} \neq 0$. In this Table, FStart is the initial frequency and FStop the end frequency. The input frequency $F$ was changed manually from FStart to FStop at steps of $0.01 \mathrm{MHz}$ at approximately 1 step per second. This way, it takes approximately 10 seconds to change the input frequency $F$ from $1.0 \mathrm{MHz}$ to $1.1 \mathrm{MHz}$, or 20 seconds to change from $1.0 \mathrm{MHz}$ to $0.8 \mathrm{MHz}$. The test results show error $\varepsilon$ is strongly affected by variations in the pulse input frequency. Even though the interpolation response is largely affected by an irregular input frequency, the final error is still lower than $0.01 \%$ as required by ISO [1].

Table 2. Results $(\mathrm{F}=1 \mathrm{MHz}$, Clock $=1 \mathrm{MHZ})$

\begin{tabular}{|c|l|l|l|l|r|r|}
\hline $\begin{array}{c}\mathbf{R} \\
\mathbf{x} 10^{2}\end{array}$ & $\begin{array}{c}|c| \\
\text { (pulses) }\end{array}$ & $\begin{array}{c}\mathbf{n} \\
\text { (pulses) }\end{array}$ & $\begin{array}{c}\text { T1 } \\
\text { (pulses) }\end{array}$ & $\begin{array}{c}\text { T2 } \\
\text { (pulses) }\end{array}$ & $\begin{array}{c}\mathbf{n} \\
\text { (pulses) }\end{array}$ & $\begin{array}{c}\boldsymbol{\varepsilon}(\%) \\
\mathbf{x} \\
10^{-5}\end{array}$ \\
\hline 20 & 19999394 & 10000 & 19999258 & 19999864 & 9999,697 & 0.000 \\
\hline 20 & 19999393 & 10000 & 19999258 & 19999864 & 9999.697 & 0.498 \\
\hline 10 & 10000075 & 10000 & 10000008 & 9999933 & 10000.075 & 0.000 \\
\hline 10 & 10000885 & 10000 & 10000818 & 9999934 & 10000.882 & 0.994 \\
\hline 1 & 1000134 & 10002 & 1000128 & 1000193 & 10001.35 & 9.994 \\
\hline 1 & 1000745 & 10008 & 1000738 & 1000794 & 10007.44 & -9.996 \\
\hline
\end{tabular}


Table 3. Results $(\mathrm{F}=1 \mathrm{MHz}$, Clock $=100 \mathrm{kHZ})$

\begin{tabular}{|c|l|l|l|l|r|r|}
\hline $\begin{array}{c}\mathbf{R} \\
\mathbf{x} 10^{2}\end{array}$ & $\begin{array}{c}\mathbf{n}_{\mathbf{A}} \\
\text { (pulses) }\end{array}$ & $\begin{array}{c}\mathbf{n} \\
\text { (pulses) }\end{array}$ & $\begin{array}{c}\text { T1 } \\
\text { (pulses) }\end{array}$ & $\begin{array}{c}\text { T2 } \\
\text { (pulses) }\end{array}$ & $\begin{array}{c}\mathbf{n}^{\mathbf{3}} \\
\text { (pulses) }\end{array}$ & $\begin{array}{c}\boldsymbol{\varepsilon} \text { (\%) } \\
\mathbf{x} \\
10^{-5}\end{array}$ \\
\hline 20 & 20000321 & 10001 & 2000019 & 2000186 & 10000,165 & 4.494 \\
\hline 20 & 20000620 & 10001 & 2000049 & 2000187 & 10000,310 & $-4,484$ \\
\hline 10 & 10000615 & 10000 & 1000055 & 999993 & 10000,620 & 5.004 \\
\hline 10 & 10000925 & 10000 & 1000086 & 999993 & 10000,930 & 5.006 \\
\hline 1 & 1000435 & 10005 & 100043 & 100049 & 10004,400 & 49.972 \\
\hline 1 & 1000764 & 10008 & 100076 & 100079 & 10007,700 & 59.951 \\
\hline
\end{tabular}

Table 4. Ramp frequency (clock $\left.=1 \mathrm{MHZ}, \varepsilon(\%)=100 \times\left(n^{\prime} \times R-n_{A}\right) / n_{A}\right)$

\begin{tabular}{|c|c|c|c|r|r|}
\hline $\begin{array}{c}\mathrm{F}_{\text {Start }} \\
(\mathrm{MHz})\end{array}$ & $\begin{array}{c}\mathrm{F}_{\text {Stop }} \\
(\mathrm{MHz})\end{array}$ & $\mathbf{R}$ & $\begin{array}{c}\mathbf{n}_{\mathbf{A}} \\
\text { (pulses) }\end{array}$ & $\begin{array}{c}\mathbf{n}^{\prime} \\
(\text { pulses })\end{array}$ & $\begin{array}{c}\boldsymbol{\varepsilon}(\%) \\
\mathbf{x} \\
10^{-5}\end{array}$ \\
\hline 1.0 & 1.1 & 1000 & 10398234 & 10398.291 & 54.944 \\
\hline 1.0 & 0.9 & 1000 & 9597962 & 9597.875 & 90.396 \\
\hline 1.0 & 0.8 & 2000 & 18107879 & 9053.724 & -238.159 \\
\hline 1.0 & 1.1 & 2000 & 22174682 & 11087.434 & 84.369 \\
\hline
\end{tabular}

\section{EXPERIMENTAL RESULTS}

In this section we verify the performance of the proposed interpolation circuitry under actual operating conditions. An oval gear type flowmeter with a resolution of four pulses per revolution was mounted to the piston prover in order to test the interpolation circuitry and control program. A data acquisition program was used to record the meter pulses output. Figure 8 shows the collected data for a total of nine pulses (2.25 revolutions). Obviously this small number of pulses is not sufficient for a meter calibration. The objective is only to verify qualitatively if the interpolation unit is working as expected under actual operating conditions. As we can see from Fig. 8, output pulses from an oval gear flowmeter are not regular. In this figure $\mathrm{Ta}=35 \mathrm{~ms}$, $\mathrm{Tb}=44 \mathrm{~ms}, \mathrm{Tc}=38 \mathrm{~ms}$ and $\mathrm{Td}=47 \mathrm{~ms}$. The average period is Tavg $=41 \mathrm{~ms}$. In this figure START indicates the time the interpolation begins and STOP the time it ends. The results obtained from the interpolation unit are as follows: $\mathrm{T} 1=328.643 \mathrm{~ms}, \mathrm{~T} 2=$ $352.662 \mathrm{~ms}, \mathrm{n}=8$ (failing edge pulses) and the calculated number of fractioned number of pulses between START and STOP is n' $=8.585$ pulses. The above results agree perfectly with the data showed in Fig. 8 demonstrating the unit works perfectly. Concerning the error between the actual value of n' and the one calculated using Equation 1 when pulses are not regular, we can observe that an error might occur depending on the START and STOP positions since the double-timing calculation assumes regular pulses. The relative error will decrease if we collect a larger number of pulses or use a meter with a larger number of pulses per revolution. The minimum number of pulses required during a meter proving is a complex issue and depends on many factors such as type of meter, flowrate stability and pulse output regularity. Meter manufacturers in conjunction with API have conducted many studies to better understand this issue [7]. Additional guidance and recommendations can be found in the standards ISO 7278-3, section 6 and API chapters 4.3, 4.6 [1-2]. 


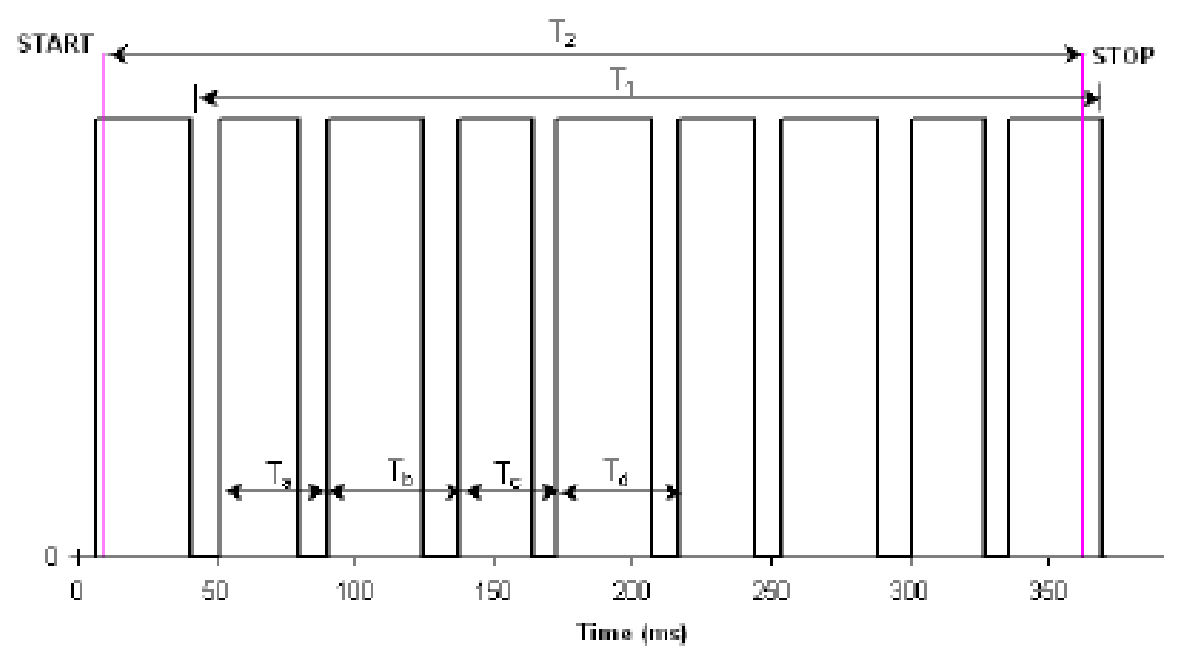

Fig. 8 Pulse output of oval gear flowmeter

\section{CONCLUSION}

The double-timing method is a reliable and widely used pulse interpolation technique that allows the calculation of the fractional number of meter pulses between two events. It allows the implementation of small volume provers with high performance and accuracy. Although the method was implemented for a small volume prover, it may also be used in other applications having pulsed outputs. The implementation of this pulse interpolation technique is straightforward and can be easily done using off-the-shelf electronic devices such as counters, timers and logical gates at a relatively low cost.

\section{REFERÊNCIAS}

[1] International Standards Organization, ISO 7278-3, 1998, Liquid hydrocarbons, Dynamic measurement, Proving Systems for Volumetric Meter, Part 3, Pulse interpolation techniques.

[2] API Manual of Petroleum Measurement Standards, 1999, Chapter 4, Proving Systems, Section 6, Pulse Interpolation, API MPMS 4.6.

[3] Yeh T. T., Aguilera J., Wright J. D., Hydrocarbon Liquid Flow Calibration Service, NIST, Special Publication, 250-1039.

[4] ANP/INMETRO Portaria Conjuntura, 2000, Regulamento Técnico de Medição de Petróleo e Gás. 
[5] Tombs M., Henry M., Zhou F., Lansangan R. M. and Reese M, 2006, High Precision Coriolis Mass Flow Measurement Applied to Small Volume Proving, Flow Measurement and Instrumentation, 17, 371-382.

[6] Yeh T. T., Espina P. I., Mattingly G.E. and Briggs N. R., An Uncertainty Analysis of a NIS Hydrocarbon Liquid Flow Calibration Facility, Proceedings of HT/FED'04, 2004 Heat Transfer/Fluids Engineering Summer Conference July 11-15 , 2004, Charlotte, North Caroline, USA.

[7] Ward P. A, Theory and Application of Pulse Interpolation to Prover Systems, Brooks Instrument, Rosemount Group, International School of Hydrocarbon Measurement, 1993.

[8] Adel S. Sedra, Kenneth C. Smith, Microelectronic circuits, New York: Oxford University Press, 2004

[9] Motorola Semiconductor Technical Data, Dual JK Flip Flop with Set and Reset, Motorola, Inc. 1995. 